\title{
Das Fellow-Programm Freies Wissen
}

Dieses Lehrbuch entstand im Rahmen der Förderung des Herausgebers durch das Fellow-Programm Freies Wissen. Im Sinne des Open-Science-Gedankens wird der Prozess der Erstellung des Lehrbuchs, von der Idee bis zur Veröffentlichung und darüber hinaus umfassend auf wikiversity.org dokumentiert. Die Dokumentation kann hier eingesehen werden:

https://de.wikiversity.org/wiki/Wikiversity:Fellow-Programm_Freies_Wis sen/Einreichungen/Erstellung_eines_OER-Lehrbuchs_zum_Verwaltungs-_und_ Verwaltungsprozessrecht

„Das Fellow-Programm Freies Wissen ist ein gemeinsames Projekt von Wikimedia Deutschland, dem Stifterverband und der VolkswagenStiftung und richtet sich an Doktoranden $(\mathrm{m} / \mathrm{w})$, Post-Docs $(\mathrm{m} / \mathrm{w})$ und Juniorprofessoren $(\mathrm{m} / \mathrm{w})$, die ihre Forschungsprozesse offen gestalten möchten. Als Partner beteiligen sich die Technische Informationsbibliothek (TIB), das Museum für Naturkunde Berlin, das Center für Digitale Systeme (CeDiS) der Freien Universität Berlin, die Niedersächsische Staats- und Universitätsbibliothek Göttingen sowie Open Knowledge Maps mit Qualifizierungsangeboten an dem Programm.

Zentrales Anliegen des Programms ist die Stärkung einer Offenen Wissenschaft, um den Wissenstransfer in die Gesellschaft und die Qualität wissenschaftlicher Forschung und Lehre zu verbessern. Transparenz, gemeinschaftliches Arbeiten und Lernen, Reproduzierbarkeit und Vergleichbarkeit wissenschaftlichen Arbeitens sind dafür elementare Voraussetzungen. Nachwuchswissenschaftler*innen aus allen Disziplinen sollen dazu befähigt werden, Prinzipien Offener Wissenschaft in die eigene wissenschaftiche Arbeit zu integrieren und gleichzeitig als Botschafter*innen andere für dieses Thema zu sensibilisieren. Gleichzeitig soll der Austausch von Wissenschaftler*innen im Bereich Offene Wissenschaft gefördert werden, um damit die schrittweise Öffnung von Wissenschaft und Forschung weiter voranzutreiben. Ein Ziel ist es daher, ein Netzwerk von Fellows, Alumni, Mentor*innen und Akteur*innen aus dem Bereich Offene Wissenschaft auf- und auszubauen, das auch über das Fellow-Programm hinaus einen intensiven Austausch und eine wirksame Zusammenarbeit im Rahmen von gemeinsamen Veranstaltungen, Publikationen und Projekten ermöglicht. Damit soll sowohl die weitere akademische Karriere der Geförderten unterstützt als auch das Thema Offene Wissenschaft kontinuierlich in die Breite getragen werden."

Quelle: https://de.wikiversity.org/wiki/Wikiversity:Fellow-Programm_Freies_ Wissen

Ә OpenAccess. (C) 2019 Nikolas Eisentraut, publiziert von De Gruyter. (cc) BY-SA Dieses Werk ist lizenziert unter der Creative Commons Attribution-ShareAlike 4.0. International. 
